\title{
Comparative Analysis of Power Electronics Topologies to Interface dc Homes with the Electrical ac Power Grid
}

\author{
Tiago J. C. Sousa \\ Industrial Electronics Deparment \\ ALGORITMI Research Centre \\ University of Minho \\ Campus de Azurem, Portugal \\ tsousa@dei.uminho.pt \\ M. J. Sepúlveda \\ Industrial Electronics Deparment \\ ALGORITMI Research Centre \\ University of Minho \\ Campus de Azurem, Portugal \\ mjs@dei.uminho.pt
}

\author{
Vítor Monteiro \\ Industrial Electronics Deparment \\ ALGORITMI Research Centre \\ University of Minho \\ Campus de Azurem, Portugal \\ vmonteiro@dei.uminho.pt \\ António Lima \\ Electrical Engineering Department \\ University of the State of \\ Rio de Janeiro \\ Brazil \\ antoniolima@uerj.br
}

\author{
Júlio S. Martins \\ Industrial Electronics Deparment \\ ALGORITMI Research Centre \\ University of Minho \\ Campus de Azurem, Portugal \\ jmartins@dei.uminho.pt \\ João L. Afonso \\ Industrial Electronics Deparment \\ ALGORITMI Research Centre \\ University of Minho \\ Campus de Azurem, Portugal \\ jla@dei.uminho.pt
}

\begin{abstract}
This paper presents a comparative analysis of power electronics topologies that can be used to interface dc homes with a $230 \mathrm{~V}, 50 \mathrm{~Hz}$ ac power grid. Dc homes represent an essential asset for smart grids, since energy storage systems and renewable energy sources, such as photovoltaic solar panels, operate in dc, as well as most of the electrical appliances used in domestic scenario. However, since the power grid operates in ac, it is necessary to convert voltage from ac to dc to properly supply a dc home. This conversion can be accomplished in several ways, with different power conversion stages. In this context, this paper analyzes three different possibilities that can be used to perform the interface between the ac power grid and a dc home: (1) ac-dc converter using a low frequency transformer; (2) ac-dc and dc-dc converters using a high frequency transformer; (3) ac-ac and ac-dc converters using a medium frequency transformer. These three possibilities are compared in terms of efficiency, total power factor and total harmonic distortion of the ac power grid. The results were obtained by means of a simulation model based on the internal parameters of the power semiconductors.
\end{abstract}

Keywords—Dc Homes, Power Electronics; Smart Grids.

\section{NOMENCLATURE}

$C_{d c} \quad$ Dc-link capacitance

$C_{o} \quad$ Dc output capacitance

$i_{g} \quad$ Ac power grid current

$i_{o} \quad$ Dc output current

$i_{x} \quad$ Transformer winding $x$ current

$L_{\text {aux }} \quad$ Transformer auxiliary inductance

$L_{g} \quad$ Ac power grid coupling inductance

$L_{l x} \quad$ Transformer winding $x$ total leakage inductance

$L_{m x} \quad$ Transformer magnetizing inductance from winding $x$

$P_{c} \quad$ Transformer core losses

$R_{c x} \quad$ Transformer core losses resistance from winding $x$

$R_{l x} \quad$ Transformer winding $x$ total resistance

$R_{o} \quad$ Dc output load resistance

$S_{n} \quad$ Transformer nominal apparent power

$v_{d c} \quad$ Dc-link voltage

$v_{g} \quad$ Ac power grid voltage

$v_{o} \quad$ Dc output voltage

$v_{x} \quad$ Transformer winding $x$ voltage

$Z_{L} \quad$ Ac power grid line impedance

$Z_{l x} \quad$ Transformer winding $x$ total leakage impedance

$Z_{s c(\%)} \quad$ Transformer short circuit impedance ratio

\section{INTRODUCTION}

The past few years witnessed an interest growth in dc power transmission and dc power grids. Despite the early establishment of ac power grids as the preferred way of power transmission and distribution, the development in power electronics turned high voltage dc (HVDC) transmission systems feasible and interesting [1]-[3]. The employment of HVDC transmission systems is even more relevant when using superconductors, eliminating voltage drops that exist in ac due both to conductors' resistance and reactance [4]-[6].

Besides the interest in HVDC transmission systems, there is an increasing concern about dc systems at the distribution and consumer levels. This is mainly justified by the paradigm of distributed generation, with microgrids being the main agent [7]-[10]. Dc power is intrinsic to energy storage systems and renewable energy sources, such as solar photovoltaic, motivating the application of dc microgrids [11]-[17]. Besides, the advantages of $\mathrm{dc}$ power can be applied to the majority of electrical appliances that are used nowadays. Despite being connected to ac power grids, a vast number of electrical loads operate in dc, i.e., representing nonlinear loads to the ac power grids. These loads are characterized by having a rectifier at its input terminals, whereby it can be advantageous to remove this converter and feed the load directly with dc power. Accordingly, the paradigm of dc homes has emerged and gained attention [19]-[21]. A study on the energy savings from supplying residential buildings with dc power can be seen in [22]. However, one important aspect of dc distribution systems and dc homes is the galvanic isolation. Since most electrical appliances operate with low voltage levels, and it is convenient to use higher voltages at the distribution level, galvanic isolation is required. Ac power transmission uses low frequency transformers, which are heavy and bulky; however, by using high frequency transformers, more compact and lightweight solutions are achieved. In [23] is analyzed and implemented a $380 \mathrm{~V}$ dc distribution system considering dc homes, using isolated dc-dc converters. The use of high frequency transformers is also the basis of the solid-state transformer (SST), which aims to replace the low frequency transformers [24], [25].

In order to evaluate the different solutions to interface a low voltage dc home to the ac power grid, this paper presents a comparative study of three different power electronics 


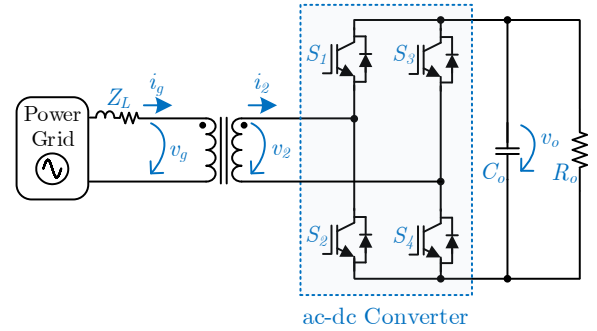

(a)

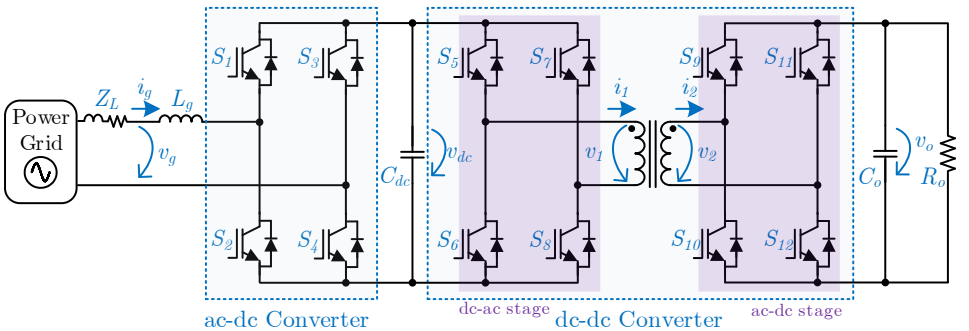

(b)
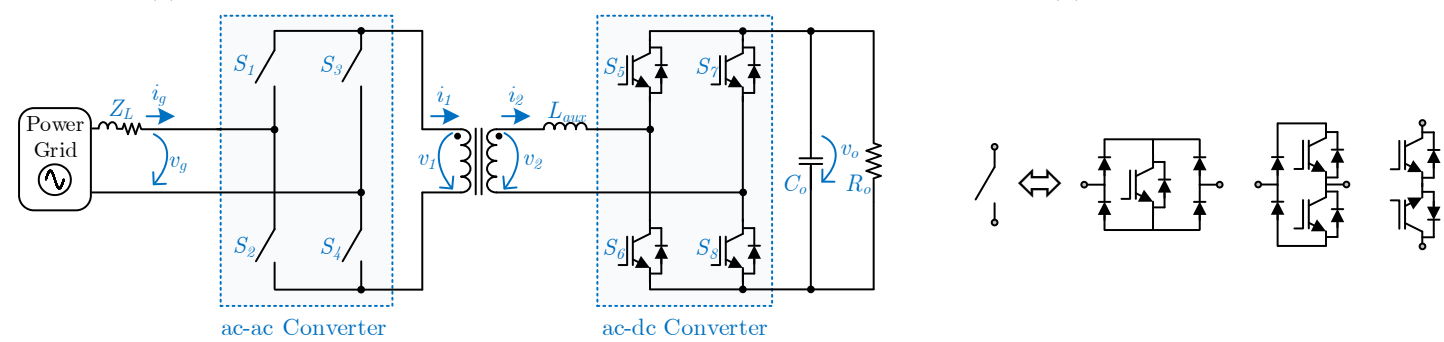

(c)

Fig. 1. Analyzed topologies for interfacing dc homes with the ac power grid: (a) ac-dc converter using a low frequency transformer; (b) ac-dc and dc-dc converters using a high frequency transformer; (c) ac-ac and ac-dc converters using a medium frequency transformer.

topologies that can perform the referred interface. The three topologies are based on: (1) ac-dc converter using a low frequency transformer; (2) ac-dc and dc-dc converters using a high frequency transformer; (3) ac-ac and ac-dc converters using a medium frequency transformer. These three cases are compared in terms of efficiency, total power factor and total harmonic distortion (THD) of the ac power grid. The results were obtained by means of a simulation model based on the internal parameters of the power semiconductors, allowing to evaluate the efficiency for the presented cases.

The paper is structured as follows: Section II presents the three analyzed topologies concerning the establishment of a dc power grid from an ac power grid; Section III presents a simulation analysis for the three considered topologies; Section IV presents a comparative analysis of the topologies; finally, Section V lists the conclusions of the developed study.

\section{ANALYZED TOPOLOGIES}

This section presents the three referred topologies considered for analysis in this paper. For all cases, it was considered an ac power grid $\left(v_{g}\right)$ of $230 \mathrm{~V}, 50 \mathrm{~Hz}$, as well as a dc output voltage $\left(v_{o}\right)$ of $48 \mathrm{~V}$. This voltage value can be found in [19] concerning dc systems. A resistive load $\left(R_{o}\right)$ was connected to the output capacitor $\left(C_{o}\right)$ in all scenarios in order to emulate the power consumption of the dc home. Due to the voltage difference between the ac power grid and the dc output, galvanic isolation was encompassed in all cases. The internal parameters of the transformers were also determined for each topology. Although in this paper it is only considered energy flow from the ac power grid to the dc home, all the topologies encompass bidirectional operation, as well as sinusoidal current and unitary power factor.

\section{A. Ac-dc Converter using a Low Frequency Transformer}

The most traditional solution for converting the ac power grid voltage into a lower dc voltage can be seen in Fig. 1 (a) (Topology 1). This topology is comprised by a low frequency transformer (in this case $50 \mathrm{~Hz}$ ) and an ac-dc converter $\left(S_{1}-S_{4}\right)$. The transformer steps down the ac power grid voltage, which is then converted to a controlled dc voltage by means of the ac-dc converter, i.e., the ac-dc converter operates as an active rectifier. It should be noted that the transformer turns ratio must be selected so that the peak secondary voltage $\left(v_{2}\right)$ is lower than the desired dc output voltage $\left(v_{o}\right)$ in order to the ac-dc converter operate as an active rectifier, controlling the voltage $v_{o}$ and maintaining a sinusoidal current consumption, as well as unitary power factor. An advantage of this topology is that it is not necessary to use an inductor to interface the ac-dc converter with the ac power grid, since the leakage inductance of the low frequency transformer is high enough to filter the ripple components produced by the converter. Besides, this is the simplest of the three analyzed topologies in this paper, using only 4 power semiconductors and the respective driver circuits; however, its main drawback is the presence of a low frequency transformer, which is heavier and bulkier compared to higher frequency solutions.

\section{B. Ac-dc and dc-dc Converters using a High Frequency Transformer}

As referred in the previous section, SSTs are a promising solution to replace low frequency transformers at the distribution level. An SST converts an ac voltage with a given frequency in another ac voltage with any value and frequency, either higher or lower than the input voltage. This is possible due to the intermediary dc stages within the SST. Therefore, in order to convert an ac voltage to a lower dc voltage, an SST without the final dc-ac stage can be used (Topology 2). Fig. 1 (b) shows the referred approach, where two power converters can be seen, namely an ac-dc $\left(S_{1}-S_{4}\right)$ and an isolated dc-dc $\left(S_{5}-S_{12}\right)$, specifically a dual active bridge, comprising two power conversion stages: a dc-ac and an ac-dc, intermediated by a high frequency transformer. The two converters share the same dc-link $\left(C_{d c}\right)$. The ac-dc converter operates in the same way as in Topology 1, i.e., as an active rectifier. On the other hand, the dc-dc converter controls the dc output voltage $\left(v_{o}\right)$. The galvanic isolation in the dc-dc converter is accomplished by a high frequency transformer, with the switches $S_{5}-S_{8}$ being subjected to a voltage $v_{d c}$ (which must be higher than the ac power grid peak voltage) and the switches $S_{9}-S_{12}$ being subjected to a voltage $v_{o}$ (which was defined as $48 \mathrm{~V}$, as aforementioned). Comparatively to Topology 1, an additional inductor is needed to interface the ac-dc converter with the ac power grid $\left(L_{g}\right)$. The main drawback of Topology 2 comparatively to Topology 1 is the higher number of power 
semiconductors and, consequently, driver circuits (12 against 4). However, the employed transformer is more lightweight and compact since it operates with a much higher frequency, as mentioned in the previous section regarding SSTs.

\section{Ac-ac and ac-dc Converters using a Medium Frequency Transformer}

In order to reduce the number of power conversions of Topology 2, namely by avoiding the intermediate dc-link, Topology 3 is considered (Fig. 1 (c)). This approach also uses two power converters, but the interface with the ac power grid is performed by an ac-ac converter $\left(S_{1}-S_{4}\right)$ instead of an ac-dc, as in Topologies 1 and 2. The second converter is an ac-dc $\left(S_{5}-S_{8}\right)$, and both converters are connected by a medium frequency transformer. The ac-ac converter is a direct matrix converter, which employs bipolar and bidirectional power switches, and it has the function of producing an appropriate voltage to be applied to the medium frequency transformer, i.e., producing an ac voltage of increased frequency $\left(v_{l}\right)$. This voltage contains both the power grid frequency and the desired medium frequency, besides the switching ripple components. The medium frequency transformer steps down this voltage $\left(v_{2}\right)$, which is then rectified by the ac-dc converter. This converter is responsible for controlling the dc output voltage $\left(v_{o}\right)$ and also the ac power grid current $\left(i_{g}\right)$ in an indirect way. The current consumed by the ac-dc converter, i.e., the transformer secondary winding current $\left(i_{2}\right)$ should be synchronized with the transformer secondary winding voltage $\left(v_{2}\right)$, containing the fundamental frequency of the power grid and the desired medium frequency. With this technique, the resulting current $i_{g}$ is mainly comprised by the fundamental frequency defined for $i_{2}$, while the ac-ac converter operates with a simple square wave modulation, multiplying the ac power grid voltage $\left(v_{g}\right)$ with a medium frequency square wave.

\section{Transformer Sizing}

This section describes the parameter determination of the transformers for the three considered topologies. Because each topology comprises a transformer with a different operating frequency, the respective internal parameters cannot be the same along the three transformers. Therefore, the same power rating was considered for the three transformers, but differences exist among them according to the defined operating frequencies. Considering nominal power rating values for transformers and keeping in mind that the dc home is supplied with a low voltage $(48 \mathrm{~V})$, the nominal value of 630 VA was selected for the transformers. The no load losses were considered to be $5 \%$ of the nominal apparent power, yielding $31.5 \mathrm{~W}$. The $X / R$ factor was defined as 5 and the short circuit impedance $\left(Z_{s c(\%)}\right)$ was established as $5 \%$. Also, in accordance with Fig. 1, the primary side was defined as the higher voltage side, i.e., the ac power grid side.

\section{1) Low Frequency Transformer}

In Topology 1, a transformer turns ratio of 10 was selected with a nominal primary voltage $\left(V_{l}\right)$ of $230 \mathrm{~V}$, resulting in a nominal secondary voltage $\left(V_{2}\right)$ of $23 \mathrm{~V}$. The nominal current can be determined as follows:

$$
I_{x}=\frac{S_{n}}{V_{x}},
$$

where $S_{n}$ is the nominal apparent power of the transformer and $I_{x}$ and $V_{x}$ the nominal current and voltage of winding $x$, respectively, with $x=1$ for the primary side and $x=2$ for the secondary side. Thus, $I_{1}=2.74 \mathrm{~A}$ and $I_{2}=27.4 \mathrm{~A}$.
In order to determine the core losses resistance reflected from winding $x\left(R_{c x}\right)$, equation (2) can be applied:

$$
R_{c_{x}}=\frac{V_{x}^{2}}{P_{c}},
$$

where $P_{c}$ is the defined value for core losses, i.e., the no load losses $(31.5 \mathrm{~W})$. From this, a value of $1.68 \mathrm{k} \Omega$ is obtained for $R_{c l}$. Considering $X / R=5$, the magnetizing inductance reflected to winding $x\left(L_{m x}\right)$ can be obtained as follows:

$$
L_{m_{x}}=\frac{5 R_{c_{x}}}{2 \pi f},
$$

where $f$ is the operating frequency of the transformer, in this case $50 \mathrm{~Hz}$. Hence, $L_{m l}=26.7 \mathrm{H}$.

In order to determine the leakage resistance and inductance of the transformer windings, the value of $Z_{s c(\%)}$ should be considered. The total leakage impedance reflected from winding $x\left(Z_{l x}\right)$ can be calculated by:

$$
Z_{l_{x}}=\frac{V_{x}}{I_{x}} Z_{s c(\%)},
$$

yielding $Z_{l l}=4.2 \Omega$. Provided that:

$$
Z_{l_{x}}=\sqrt{R_{l_{x}}^{2}+X_{l_{x}}^{2}}
$$

and $X / R=5$, a value of $0.823 \Omega$ is obtained for the total leakage resistance reflected to the primary winding $\left(R_{l l}\right)$. The total leakage inductance reflected to the primary winding $\left(L_{l 1}\right)$ can be determined with an equation similar to (3), using $L_{l 1}$ instead of $L_{m x}$ and $R_{l l}$ instead of $R_{c l}$, resulting in $13.1 \mathrm{mH}$. This justifies the absence of a grid coupling inductor in Topology 1 , since this value is already reasonable for the connection of the ac-dc converter to the ac power grid.

\section{2) High Frequency Transformer}

Topology 2 employs a high frequency transformer in a dual active bridge dc-dc converter. In order to avoid the use of external inductors in this converter, the operating frequency was established as $100 \mathrm{kHz}$. As it can be seen in the literature, the most common and flexible method for power transfer in this type of converter is the phase shift modulation and its derivatives, i.e., dual phase shift or triple phase shift. With this technique, the transformer should have a turns ratio close to the ratio of the dc-link voltages of each side of the converter in order to minimize the reactive power in the converter and transformer. The primary side dc-link voltage $\left(v_{d c}\right)$, which is controlled by the ac-dc converter, should have an average value higher than the ac power grid peak voltage, so that the ac power grid current $\left(i_{g}\right)$ can be controlled. Therefore, a reference value of $400 \mathrm{~V}$ was chosen for $v_{d c}$. The secondary side dc-link voltage $\left(v_{o}\right)$ is the dc output voltage, i.e., the dc home voltage $(48 \mathrm{~V})$. Taking both voltages in consideration, the transformer turns ratio was established as 8 . The resulting parameters for the transformer can be seen in Table I.

\section{3) Medium Frequency Transformer}

Topology 3 employs a medium frequency transformer interfacing the ac-ac and ac-dc converters. Because the ac-dc converter must absorb a current with the same waveform as the transformer secondary voltage, which consists in the ac power grid voltage multiplied by a square wave, its switching frequency has to be several times higher than the defined medium frequency for the modulation and, consequently, the transformer. In this paper, a frequency of $1 \mathrm{kHz}$ was chosen.

The operation principle of the ac-dc converter in Topology 3 is similar to that of Topology 1, i.e., controlling the dc output voltage to the reference value of $48 \mathrm{~V}$. Thus, a 


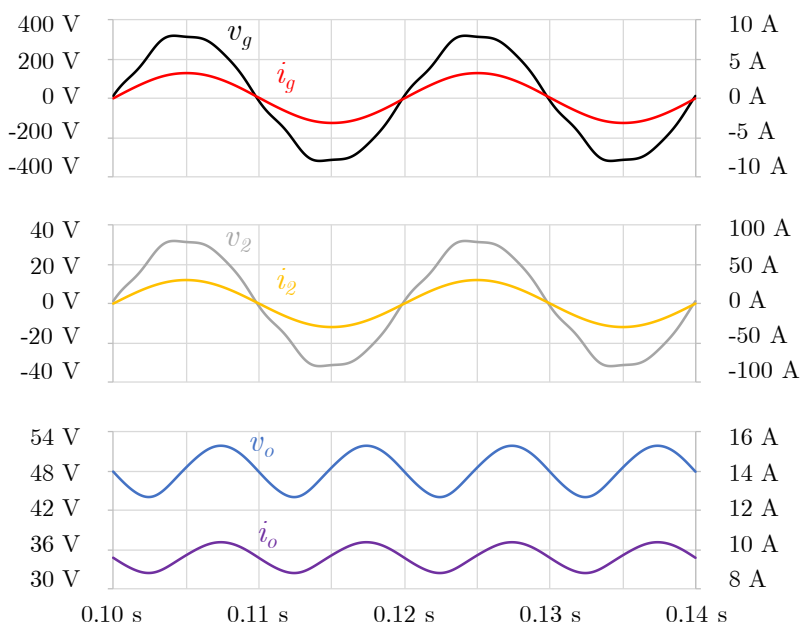

Fig. 2. Simulation results obtained with Topology 1: ac power grid voltage $\left(v_{g}\right)$ and current $\left(i_{g}\right)$; transformer secondary winding voltage $\left(v_{2}\right)$ and current $\left(i_{2}\right)$; dc output voltage $\left(v_{o}\right)$ and current $\left(i_{o}\right)$.

turns ratio of 10 was also chosen for the medium frequency transformer, with the nominal values of voltage, current and resistance being the same as the transformer in Topology 1. The only difference from the low frequency transformer resides in the inductance values, since the operating frequency is different. Table I shows the referred parameters. Besides, as it can be seen in Fig. 1 (c), there is an auxiliary inductor $\left(L_{\text {aux }}\right)$ placed in the secondary winding due to the insufficient value of $L_{l 2}(6.55 \mu \mathrm{H})$. In this paper, it was considered $L_{a u x}=50 \mu \mathrm{H}$.

TABle I. TRANSFORMER DESIGN PARAMETERS.

\begin{tabular}{|c|c|c|c|}
\hline PARAMETER & TOPOLOGY 1 & TOPOLOGY 2 & TOPOLOGY 3 \\
\hline$V_{l}$ & $230 \mathrm{~V}$ & $400 \mathrm{~V}$ & $230 \mathrm{~V}$ \\
\hline$V_{2}$ & $23 \mathrm{~V}$ & $50 \mathrm{~V}$ & $23 \mathrm{~V}$ \\
\hline$I_{1}$ & $2.74 \mathrm{~A}$ & $1.58 \mathrm{~A}$ & $2.74 \mathrm{~A}$ \\
\hline$I_{2}$ & $27.4 \mathrm{~A}$ & $12.6 \mathrm{~A}$ & $27.4 \mathrm{~A}$ \\
\hline$f$ & $50 \mathrm{~Hz}$ & $100 \mathrm{kHz}$ & $1 \mathrm{kHz}$ \\
\hline$L_{l 1}$ & $13.1 \mathrm{mH}$ & $19.82 \mu \mathrm{H}$ & $655 \mu \mathrm{H}$ \\
\hline$R_{l l}$ & $0.823 \Omega$ & $2.49 \Omega$ & $0.823 \Omega$ \\
\hline$L_{m l}$ & $26.7 \mathrm{H}$ & $40.4 \mathrm{mH}$ & $1.34 \mathrm{H}$ \\
\hline$R_{c l}$ & $1.68 \mathrm{k} \Omega$ & $5.08 \mathrm{k} \Omega$ & $1.68 \mathrm{k} \Omega$ \\
\hline
\end{tabular}

\section{Simulation ANALYSIS}

This section presents the obtained simulation results of the three topologies individually. The simulation models were developed in the software PSIM v9.1 from PowerSim and the database models of power semiconductors were used, so that the topologies can be evaluated in terms of efficiency. For a proper comparison, it were used common parameters to the three topologies, as listed in Table II. The used semiconductors were insulated gate bipolar transistors (IGBTs) model IXYS IXGH40N60C2 with the respective antiparallel diodes. The power semiconductors are switched with a unipolar pulse-width modulation (PWM) in all the ac-dc converters. In addition, as it can be seen in Table I, four values of output load $\left(R_{o}\right)$ were used, so that the comparative analysis can be evaluated for different values of output power. For the present section regarding the individual results of the topologies, however, only the load $R_{o}=5 \Omega$ was used.

\section{A. Ac-dc Converter using a Low Frequency Transformer}

This section shows the results obtained with Topology 1 . Fig. 2 depicts the main waveforms of voltage and current within this topology, namely the ac power grid voltage $\left(v_{g}\right)$

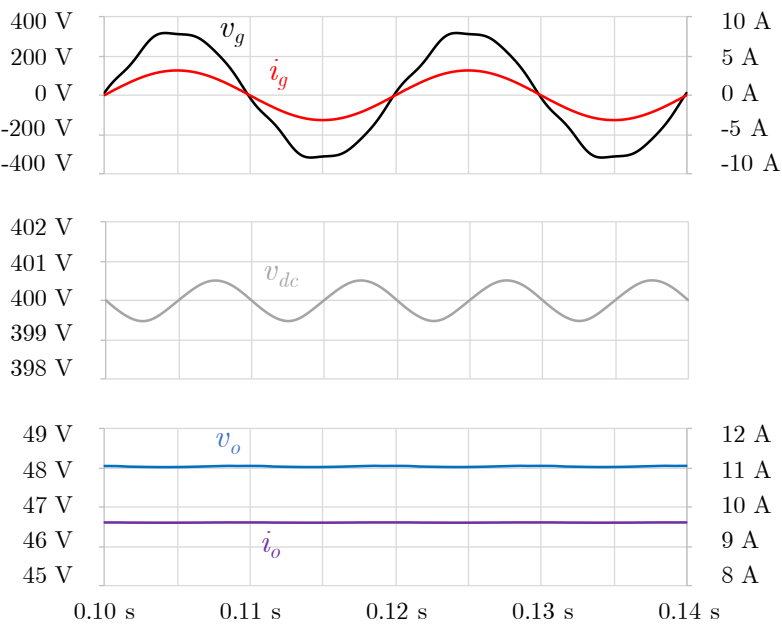

Fig. 3. Simulation results obtained with Topology 2: ac power grid voltage $\left(v_{g}\right)$ and current $\left(i_{g}\right)$; dc-link voltage $\left(v_{d c}\right)$; dc output voltage $\left(v_{o}\right)$ and current $\left(i_{o}\right)$.

TABle II. General Parameters of the Simulation Model.

\begin{tabular}{|c|c|}
\hline PARAMETER & VALUE \\
\hline ac Power Grid $\left(V_{g}\right)$ & $230 \mathrm{~V}, 50 \mathrm{~Hz}$ \\
\hline Line Impedance $\left(Z_{L}\right)$ & $0.1 \Omega, 10 \mu \mathrm{H}$ \\
\hline Capacitors $\left(C_{o}, C_{d c}\right)$ & $4 \mathrm{mF}$ \\
\hline Sampling Frequency & $100 \mathrm{kHz}$ \\
\hline Switching Frequency & $100 \mathrm{kHz}$ \\
\hline Output Load $\left(R_{o}\right)$ & $\{5,10,20,40\} \Omega$ \\
\hline
\end{tabular}

and the respective absorbed current $\left(i_{g}\right)$, which are also the transformer primary winding voltage $\left(v_{1}\right)$ and current $\left(i_{1}\right)$, respectively; the transformer secondary winding voltage $\left(v_{2}\right)$ and current $\left(i_{2}\right)$; and the dc output waveforms $\left(v_{o}\right.$ and $\left.i_{o}\right)$. As referred, the output load $\left(R_{o}\right)$ is $5 \Omega$, which is the lowest of the listed values in Table II and results in an output power of $460 \mathrm{~W}$. In this case, the ripple in $v_{o}$ is around $2.5 \mathrm{~V}$ peak with a frequency of $100 \mathrm{~Hz}$, with its average value being controlled to $48 \mathrm{~V}$, as expected. Besides, the current $i_{g}$ is sinusoidal and in-phase with the voltage $v_{g}$, meaning a low value of THD $(1.72 \%)$ and a high total power factor (practically unitary).

\section{B. Ac-dc and dc-dc Converters using a High Frequency Transformer}

This section depicts the main voltage and current waveforms of Topology 2. In this topology, a grid coupling inductor $\left(L_{g}\right)$ should be used, since no transformer is used to connect the ac-dc converter to the power grid. For this purpose, an inductance value of $2 \mathrm{mH}$ was used.

The referred results are shown in Fig. 3. Once again, the ac power grid waveforms $\left(v_{g}\right.$ and $\left.i_{g}\right)$ and the dc output waveforms $\left(v_{o}\right.$ and $i_{o}$ ) can be seen. Similarly to the previous result, the current $i_{g}$ is sinusoidal and in-phase with the voltage $v_{g}$, but its THD is slightly higher $(2.50 \%)$, since the leakage inductance of the low frequency transformer from Topology 1 is higher than the inductance of $L_{g}$. However, using an inductance to match the value of Topology 1 has no practical interest, especially with a switching frequency of $100 \mathrm{kHz}$. On the other hand, it can be seen that the dc output voltage $\left(v_{o}\right)$ has a much lower ripple (around $10 \mathrm{mV}$ ) than the previous topology for the same output load. This figure also shows the dc-link voltage that interfaces both converters $\left(v_{d c}\right)$, controlled to the established reference average value of $400 \mathrm{~V}$.

Because the leakage inductance of the high frequency transformer is small, the phase shift modulation can lead to 


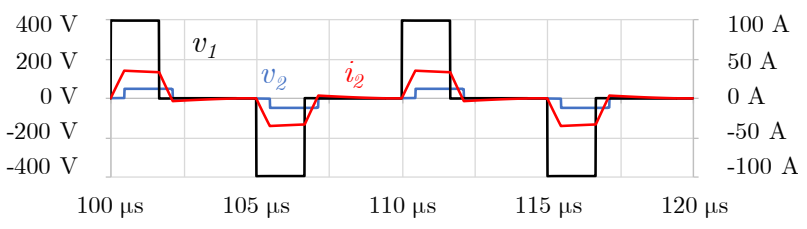

Fig. 4. Simulation results obtained with Topology 2: Detail of the voltages in the primary $\left(v_{1}\right)$ and secondary $\left(v_{2}\right)$, and current in the secondary $\left(i_{2}\right)$ winding of the transformer.

high values of reactive power and, consequently, large values of circulating current in the transformer windings and in the power semiconductors. In order to minimize this effect, a dual phase shift modulation was used. Fig. 4 shows the waveforms regarding the transformer, namely the applied voltages to the primary $\left(v_{1}\right)$ and secondary $\left(v_{2}\right)$ windings, and the current in the secondary winding $\left(i_{2}\right)$. The current in the primary winding is not shown because it has the same waveform of $i_{2}$ with a value 8 times lower, according to the transformer turns ratio. It can be seen that the voltage $v_{1}$ is leading $v_{2}$, meaning that energy is being transferred from the primary to the secondary winding or, in other words, from $v_{d c}$ to $v_{o}$. Besides, it can be seen that both voltages contain three levels $\left(+v_{d c}, 0,-v_{d c}\right.$ for $v_{1}$ and $+v_{o}, 0,-v_{o}$ for $v_{2}$ ), as expected for the dual phase shift modulation.

\section{Ac-ac and ac-dc Converters using a Medium Frequency Transformer}

This section depicts the main voltage and current waveforms of Topology 3 . As aforementioned, an auxiliary inductor $\left(L_{a u x}\right)$ should be used in series with one of the transformer windings because of the insufficient leakage inductance value of the medium frequency transformer. For this purpose, an inductance value of $50 \mu \mathrm{H}$ was used, with the inductor being placed in the secondary winding due to the fact of being the lowest impedance transformer side. This inductor could also be connected in the primary winding, but its value would be $5 \mathrm{mH}$ due to the transformer turns ratio.

Regarding the ac-ac converter, as shown in Fig. 1 (c), the bipolar and bidirectional power switches can be comprised by one of the three configurations shown in the same figure: one switch and four diodes; two series switches and two diodes; two anti-series switches (common emitter configuration) with the respective anti-parallel diodes. In this paper, the common emitter configuration was chosen, since the commercially available power switches contain an anti-parallel diode. Hence, the ac-ac converter is composed of 8 power switches. It should be noted that this converter is not switched at $100 \mathrm{kHz}$ but at $1 \mathrm{kHz}$ due to the square wave modulation, as previously referred.

The referred results for Topology 3 are shown in Fig. 5. Once again, the ac power grid waveforms $\left(v_{g}\right.$ and $\left.i_{g}\right)$ and the dc output waveforms $\left(v_{o}\right.$ and $\left.i_{o}\right)$ can be seen. Additionally, this figure also shows the voltage $\left(v_{2}\right)$ and current $\left(i_{2}\right)$ in the secondary winding of the transformer. Similarly to the previous results, the current $i_{g}$ is in-phase with the voltage $v_{g}$, but has a reasonably higher THD (40.15\%). This high value is due to large current spikes that are a consequence of fast variations in the voltage applied to the transformer $\left(v_{l}\right)$, which is the result of the square wave modulation of the ac-ac converter. Besides, the current spikes present in $i_{g}$ tend to be higher for higher values of $L_{a u x}$, whereby this inductance cannot be too high nor too small, so that the current $i_{2}$ can be properly synthesized. As it can be seen in the figure, the current $i_{2}$ is synchronized with the voltage $v_{2}$, being verified the proper operation of the ac-dc converter. In terms of $\mathrm{dc}$

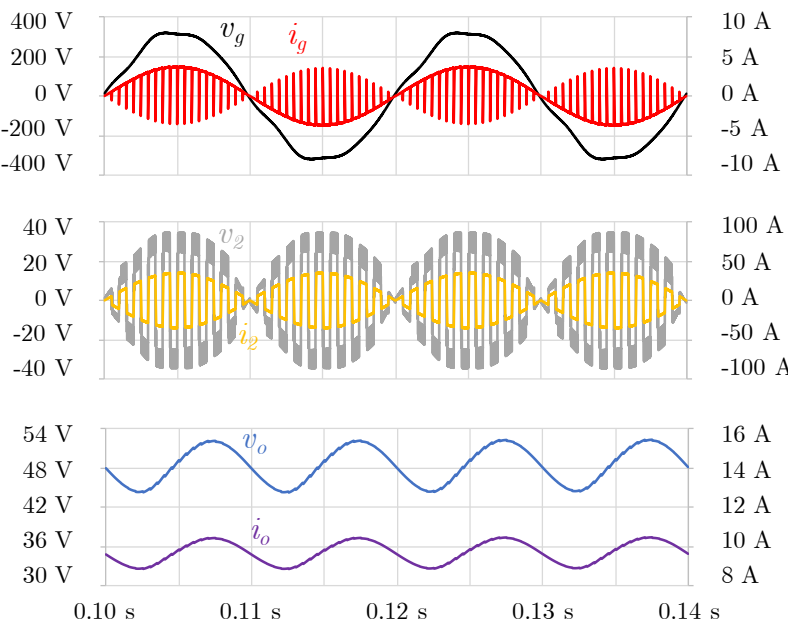

Fig. 5. Simulation results obtained with Topology 3: ac power grid voltage $\left(v_{g}\right)$ and current $\left(i_{g}\right)$; transformer secondary winding voltage $\left(v_{2}\right)$ and current $\left(i_{2}\right)$; dc output voltage $\left(v_{o}\right)$ and current $\left(i_{o}\right)$.

output voltage $\left(v_{o}\right)$, it can be seen the average value of $48 \mathrm{~V}$ and a ripple component of $100 \mathrm{~Hz}$, plus a small $1 \mathrm{kHz}$ component. The total peak ripple is approximately $3 \mathrm{~V}$.

\section{COMPARATIVE ANALYSIS}

After analyzing the individual simulation results of the three topologies for a single operating condition, this section presents a comparison of the three topologies in terms of total power factor, THD and efficiency for a given range of output power values, i.e., for the output load values referred in Table II $\quad(5 \Omega, \quad 10 \Omega, 20 \Omega, 40 \Omega, \quad$ corresponding approximately to power values of $460 \mathrm{~W}, 230 \mathrm{~W}, 115 \mathrm{~W}$ and $58 \mathrm{~W}$, respectively). These results can be seen in Fig. 6 .

In terms of total power factor, it can be seen that the best results are obtained with Topology 1 and 2, with the highest values being attained for the higher output power values. In fact, Topology 1 is slightly better than Topology 2 in light load conditions, but both values are still high (0.992 against 0.982). In contrast, Topology 3 presents a different variation, with the total power factor decreasing with the output power. Nevertheless, the obtained values are still close to unity, with the lowest registered value being 0.928 for Topology 3 .

In terms of THD of the ac power grid current $\left(i_{g}\right)$, the results are similar to the total power factor case. The best performance is achieved with Topology 1 , as it could be seen in the previous section. For Topology 1 and 2, the THD reduces with the output power, as expected, but with Topology 3 it presents the opposite variation. This is verified because the current spikes in $i_{g}$ are higher for higher values of output power, with the THD being $17.13 \%$ for $58 \mathrm{~W}$ and $40.15 \%$ for $460 \mathrm{~W}$.

Lastly, the three topologies were also compared in terms of efficiency, i.e., concerning the input (ac power grid side) and output (dc home side) values of active power. In this case, the best results were obtained with Topology 2. The highest efficiency value was obtained for both Topology 1 and 2 for $460 \mathrm{~W}(89.9 \%)$, but, for the considered operating range, Topology 2 presents the best results, since its lowest efficiency value is $83.9 \%$ for $58 \mathrm{~W}$, while Topology 1 has only an efficiency of $63.9 \%$ for the same output power. In this case, Topology 3 presents the same behavior as the other topologies, with the efficiency incrementing with the output power, and its obtained values are close to those of Topology 1 , varying between $64.3 \%$ and $88.5 \%$. 


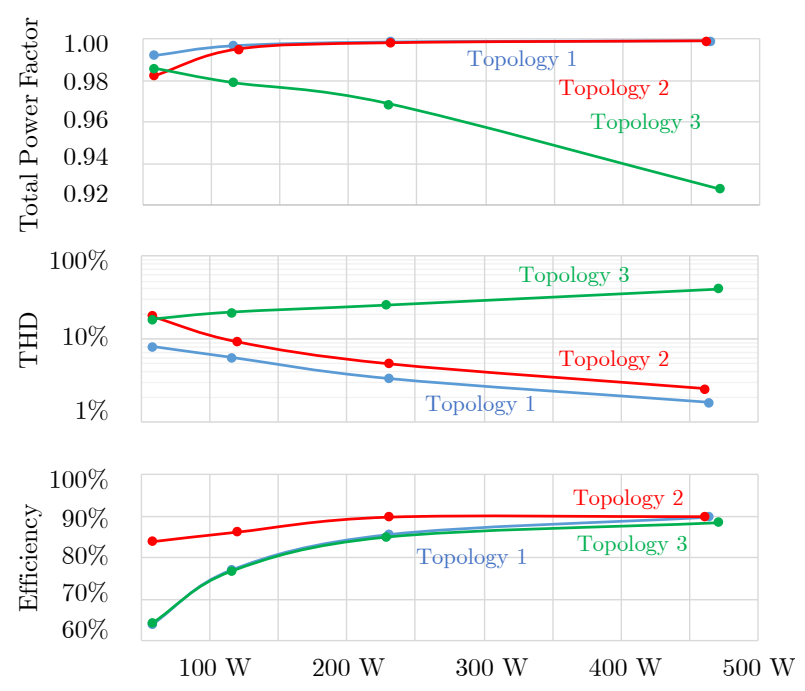

Fig. 6. Total power factor, THD and efficiency for the three topologies, with respect to the output power, obtained in simulation.

\section{CONCLUSIONS}

This paper presented a comparison of three power electronics topologies to interface a dc home with a $230 \mathrm{~V}$, $50 \mathrm{~Hz}$ ac power grid. The considered topologies were: (1) ac-dc converter using a low frequency transformer; (2) ac-dc and dc-dc converters using a high frequency transformer; (3) ac-ac and ac-dc converters using a medium frequency transformer. The results were obtained by means of a simulation model considering the database model of the power semiconductors. The comparison was performed in terms of total power factor in of the ac power grid, total harmonic distortion (THD) of the current in the ac power grid, and efficiency. It could be seen that the best results in terms of total power factor and THD were obtained for Topology 1 , with only a slight difference from Topology 2 . On the other hand, the best efficiency results were obtained for Topology 2, taking into consideration the analyzed power range. Despite the good results of Topology 1 , it should be noted that this approach employs a low frequency transformer, which is heavier and bulkier than the high frequency and medium frequency transformers of the other topologies. Therefore, Topology 2 presents a better compromise between performance and volume/weight. It should be mentioned that Topology 3 aimed at reducing the number of power conversion stages of Topology 2, but the obtained results showed no advantages, neither in terms of performance or physical size.

\section{ACKNOWLEDGMENTS}

This work has been supported by FCT - Fundação para a Ciência e Tecnologia within the Project Scope: UID/CEC/00319/2019. This work has been supported by FCT Project DAIPESEV PTDC/EEI-EEE/30382/2017, and by the FCT Project newERA4GRIDs PTDC/EEI-EEE/30283/2017. Mr. Tiago Sousa is supported by the doctoral scholarship SFRH/BD/134353/2017 granted by FCT.

\section{REFERENCES}

[1] IEEE Standards Association, "Recommended Practice for Determination of Power Losses in High- Voltage Direct-Current (HVDC) Converter Stations.," IEEE Std 1158-1991, pp. 1-58, 1991.
[2] IEEE Standards Association, "IEEE Guide for Commissioning HighVoltage Direct-Current (HVDC) Converter Stations and Associated Transmission Systems," IEEE Std 1378-1997, pp. 1-25, 1997.

[3] IEEE Standards Association, "IEEE Guide for the Evaluation of the Reliability of HVDC Converter Stations," IEEE Std 1240-2000, pp. 1$61,2000$.

[4] B. Malek and B. K. Johnson, "Branch Current Control on a Superconducting DC Grid," IEEE Trans. Appl. Supercond., vol. 23, no. 3, pp. 5401005-5401005, Jun. 2013.

[5] A. Marian, S. Holé, F. Lesur, M. Tropeano, and C. E. Bruzek, "Validation of the superconducting and insulating components of a high-power HVDC cable," IEEE Elect. Insul. Mag., vol. 34, no. 1, pp. 26-36, Jan. 2018.

[6] S.-M. Baek, H.-J. Kim, J.-W. Cho, and H.-S. Ryoo, "Cryogenic Electrical Insulation Characteristics of Solid Insulator for the HVDC HTS Cable," IEEE Trans. Appl. Supercond., vol. 28, no. 4, pp. 1-4, Jun. 2018.

[7] R. H. Lasseter, "MicroGrids," in 2002 IEEE Power Engineering Society Winter Meeting. Conference Proceedings (Cat. No.02CH37309), 2002, vol. 1, pp. 305-308.

[8] J. A. P. Lopes, C. L. Moreira, and A. G. Madureira, "Defining Control Strategies for MicroGrids Islanded Operation," IEEE Trans. Power Syst., vol. 21, no. 2, pp. 916-924, May 2006.

[9] J. de Matos, F. e Silva, and L. Ribeiro, "Power Control in AC Isolated Microgrids with Renewable Energy Sources and Energy Storage Systems," IEEE Trans. Ind. Electron., vol. 62, no. 6, pp. 1-1, 2014.

[10] Y. Han, H. Li, P. Shen, E. A. A. Coelho, and J. M. Guerrero, "Review of Active and Reactive Power Sharing Strategies in Hierarchical Controlled Microgrids," IEEE Trans. Ind. Electron., vol. 32, no. 3, pp. 2427-2451, Mar. 2017.

[11] A. Kwasinski, "Quantitative Evaluation of DC Microgrids Availability: Effects of System Architecture and Converter Topology Design Choices," IEEE Trans. Power Electron., vol. 26, no. 3, pp. 835-851, Mar. 2011.

[12] P. Fairley, "Edison vindicated [Update]," IEEE Spectrum, vol. 48, no. 2, pp. 14-16, Feb. 2011.

[13] P. Fairley, "DC Versus AC: The Second War of Currents Has Already Begun [In My View]," IEEE Power Energy Mag., vol. 10, no. 6, pp. 103104, Nov. 2012

[14] T. Dragicevic, J. C. Vasquez, J. M. Guerrero, and D. Skrlec, "Advanced LVDC Electrical Power Architectures and Microgrids: A step toward a new generation of power distribution networks.," IEEE Electrific. Mag., vol. 2 , no. 1, pp. 54-65, Mar. 2014.

[15] D. Jovcic, M. Taherbaneh, J.-P. Taisne, and S. Nguefeu, "Offshore DC Grids as an Interconnection of Radial Systems: Protection and Control Aspects," IEEE Trans. Smart Grid, vol. 6, no. 2, pp. 903-910, Mar. 2015.

[16] F. Wang, Z. Lei, X. Xu, and X. Shu, "Topology Deduction and Analysis of Voltage Balancers for DC Microgrid," IEEE Trans. Emerg. Sel. Topics Power Electron., vol. 5, no. 2, pp. 672-680, Jun. 2017.

[17] D. Kumar, F. Zare, and A. Ghosh, "DC Microgrid Technology: System Architectures, AC Grid Interfaces, Grounding Schemes, Power Quality, Communication Networks, Applications, and Standardizations Aspects," IEEE Access, vol. 5, pp. 12230-12256, 2017.

[18] B. T. Patterson, "DC, Come Home: DC Microgrids and the Birth of the 'Enernet,"' IEEE Power Energy Mag., vol. 10, no. 6, pp. 60-69, 2012.

[19] E. Rodriguez-Diaz, J. C. Vasquez, and J. M. Guerrero, "Intelligent DC Homes in Future Sustainable Energy Systems: When efficiency and intelligence work together," IEEE Consum. Electron. Mag., vol. 5, no. 1, pp. 74-80, Jan. 2016.

[20] A. Ghazanfari and Y. A.-R. I. Mohamed, "Decentralized Cooperative Control for Smart DC Home with DC Fault Handling Capability," IEEE Trans. Smart Grid, vol. 9, no. 5, pp. 1-1, 2017.

[21] N. Sasidharan and J. G. Singh, "A Novel Single-Stage Single-Phase Reconfigurable Inverter Topology for a Solar Powered Hybrid AC/DC Home," IEEE Trans. Ind. Electron., vol. 64, no. 4, pp. 2820-2828, 2017.

[22] V. Vossos, K. Garbesi, and H. Shen, "Energy savings from direct-DC in U.S. residential buildings," Energy and Buildings, vol. 68, no. PARTA, pp. 223-231, Jan. 2014.

[23] M. H. Ryu, H. S. Kim, J. W. Baek, H. G. Kim, and J. H. Jung, "Effective test bed of 380-V DC distribution system using isolated power converters," IEEE Trans. Ind. Electron., vol. 62, no. 7, pp. 4525-4536, 2015.

[24] J. E. Huber and J. W. Kolar, "Solid-State Transformers," IEEE Ind. Electron. Mag., vol. 10, no. 3, pp. 19-28, 2016.

[25] X. Yu, X. She, X. Zhou, and A. Q. Huang, "Power management for DC microgrid enabled by solid-state transformer," IEEE Trans. Smart Grid, vol. 5, no. 2, pp. 954-965, 2014. 